\title{
Response of a climate model to tidal mixing parameterization under present day and Last Glacial Maximum conditions
}

\author{
Álvaro Montenegro, ${ }^{\mathrm{a},}{ }^{*}$ Michael Eby, ${ }^{\mathrm{a}}$ Andrew J. Weaver ${ }^{\mathrm{a}}$ \\ and Steven R. Jayne ${ }^{b}$

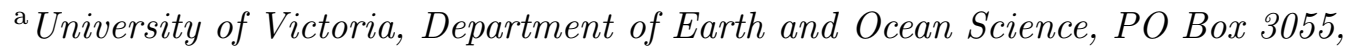 \\ Stn CSC, Victoria, BC, V8W 3P6, CA \\ ${ }^{\mathrm{b}}$ Woods Hole Oceanographic Institution, Woods Hole, MA, USA
}

\begin{abstract}
Experiments with a climate model were conducted under present day and last glacial maximum conditions in order to examine the model's response to a vertical mixing scheme based on internal tide energy dissipation. The increase in internal tide energy flux caused by a $\sim 120 \mathrm{~m}$ reduction in sea level had the expected effect on diffusivity values, which were higher under lower sea level conditions. The impact of this vertical diffusivity change on the Atlantic meridional overturning is not straightforward and no clear relationship between diffusivity and overturning is found. There exists a weak positive correlation between overturning and changes to the power consumed by vertical mixing. Most of the climatic response generated by sea level change was not related to alterations in the internal tide energy flux but rather to the direct change in sea level itself.
\end{abstract}

Key words: tidal mixing, last glacial maximum, sea level change 


\section{Introduction}

Observations point to large spatial heterogeneity in ocean vertical mixing (Wunsch and Ferrari (2004)) and to the fact that internal tides are an important source of mechanical energy for this mixing (Egbert and Ray (2000); Jayne and St. Laurent (2001)). Also, several studies show that mixing rates increase over areas where internal tides are generated (Ledwell et al. (2000); Moum et al. (2002); Nash et al. (2004); Sloyan (2005). In most ocean models, vertical mixing is represented in a way that does not take into account this spatial variability nor the relationship between mixing and tides. While a more physically based mixing parameterization would likely benefit any model, it is especially pertinent to simulations of future or past climate states, as no direct estimates of mixing are available for these cases.

One potentially important and overlooked process in different climate states is the change in mixing associated with sea level variations. The amount of tidal energy available for mixing by internal tides in the deep ocean is inversely related to amount of tidal energy consumed by mixing in shallow areas. As the area of inundated continental shelf changes, so should deep ocean tidal mixing. An increase in internal tide dissipation in the deep ocean has been found in a modelling study of the Last Glacial Maximum (LGM) (Egbert et al. (2004)).

We discuss the implementation of a tidal mixing scheme (Jayne and St. Laurent (2001)) in the University of Victoria Earth System Climate Model (UVic ESCM, Weaver et al. (2001)) under LGM conditions (including a reduction

\footnotetext{
* Corresponding author.
}

phone:(250) 472-4002

Email address: alvaro@ocean.seos.uvic.ca (Álvaro Montenegro,). 
in sea level). We also present results from present day simulations that used the mixing scheme and compare these to experiments conducted with different Bryan-Lewis type vertical mixing profiles (Bryan and Lewis (1979)).

In the adopted parameterization, tidal mixing depends on bottom roughness and is spatially heterogeneous. Nonetheless, we do not center the analyses at local or regional scales, but instead focus on the large scale effects of the changes to tidal mixing caused by sea level change, especially on alterations of the Atlantic meridional overturning circulation (MOC), global water mass distribution and global vertical diffusivity $\left(k_{v}\right)$ profile.

\section{Methods}

\subsection{UVic ESCM}

The UVic ESCM is a model of intermediate complexity used in previous studies of present day (PD) and LGM climate and ocean circulation (Weaver et al. (2001); Schmittner et al. (2002)). All components of the model have horizontal resolution of $1.8^{\circ} \times 3.6^{\circ}$. The ocean component is version 2.2 of the GFDL Modular Ocean Model (Pacanowski (1996)) with 19 vertical levels. Grid box thickness varies from $50 \mathrm{~m}$ at the surface to about $500 \mathrm{~m}$ at depths greater than $5000 \mathrm{~m}$. The effect of eddies was parameterized based on the GentMcWilliams mixing scheme with both isopycnal and thickness diffusivity set at $4 \times 10^{6}$ (Gent and McWilliams (1990); Gent et al. (1995)).

Sea ice is simulated by a dynamic-thermodynamic sea ice model and continental ice sheet area extend and height were prescribed according to Peltier 
(2004). Land cover is simulated by the Hadley Centre dynamical global vegetation model (Meissner et al. (2003)). The UVic ESCM atmosphere is a simple energy-moisture balance model.

\subsection{Mixing parameterization}

The vertical diffusivity $\left(k_{v}\right)$ of the tidal mixing experiments comes from the parameterization proposed by St. Laurent et al. (2002), which is based on the energy flux from barotropic tides into the internal wave field (Jayne and St. Laurent (2001)). This parameterization was implemented in the UVic ESCM following the scheme developed by Simmons et al. (2004) and used in other studies (Saenko (2006); Schmittner et al. (2005)).

The barotropic tide energy flux into the internal wave field per unit area is given by:

$$
E(x, y)=\frac{1}{2} \rho_{o} N_{b} k h^{2}<\mathbf{u}^{2}>
$$

where $\rho_{o}$ is a reference sea water density, $N_{b}$ is the climatological buoyancy frequency at the sea floor, $k$ and $h$ are scales for bottom roughness wavenumber and amplitude respectively and $\mathbf{u}$ is the barotropic tidal velocity (Jayne and St. Laurent (2001)). For simplicity, from now on the text will refer to the barotropic tide energy flux into the internal wave field as either $E$ or "energy flux".

Velocity values $(\mathbf{u})$ come from the hydrodynamic tide model with $0.5^{\circ}$ by $0.5^{\circ}$ horizontal resolution described by Jayne and St. Laurent (2001). For this study, the model's meridional limits were extended from the original $\pm 72^{\circ}$ 
to $\pm 82^{\circ}$. The model is run under two configurations: present day sea level (defined as high sea level) and present day sea level minus 120 m (defined as low sea level) to provide two distinct energy flux fields. Sea level change is accomplished by a change in the sea floor depth of the whole ocean. The goal is to generate changes in $E$ associated with a change in sea level comparable to the one that occurred between the PD and the LGM. Changes in energy flux due to differences in LGM bottom density or topography are not considered and $\rho_{0}, N_{b}, k$ and $h^{2}$ are the same for both high sea level and low sea level $E$ estimates.

The resulting energy flux fields have large spatial heterogeneity, with larger fluxes over regions of increased roughness (Fig.1, top). The tide model reproduces the expected increase in $E$ associated with the lower sea level of the LGM configuration, where less tidal energy is dissipated over the shelves, consequently increasing barotropic tide speeds in the deep ocean and the amount of energy transferred into internal waves in this region (Fig.1, center). Both PD and LGM energy flux fields were generated with a $0.5^{\circ}$ resolution. Prior to their insertion into the UVic ESCM, the fields were interpolated to the coarser grid of the climate model. The total energy fluxes (spatial integrals) were preserved by the interpolation (Fig.1, bottom). As the tide model did not extend beyond $82^{\circ} \mathrm{N}$ there were no $E$ values available poleward of this latitude. In the Arctic area north of $82^{\circ} \mathrm{N}$ diffusivity is set to $1 \mathrm{~cm}^{2} \mathrm{~s}^{-1}$ for the whole water column.

The energy flux provided by the tide model is used in the UVic ESCM to calculate $k_{v}$ by:

$$
k_{v}=k_{o}+\frac{q \Gamma E(x, y) F(z)}{\rho N^{2}}
$$


where $k_{o}$ is the background vertical diffusivity (set to $0.2 \mathrm{~cm}^{2} \mathrm{~s}^{-1}$ ), $q$ the tidal dissipation efficiency (set to $1 / 3$ ), and $\Gamma$ the mixing efficiency (set to 0.2 ), $\rho$ is local density and $N$ local buoyancy frequency. The vertical structure function for $k_{v}, F(z)$, is based on microstructure observations and defined by:

$$
F(z)=\frac{e^{-\frac{H+z}{\zeta}}}{\zeta\left(1-e^{-\frac{H}{\zeta}}\right)}
$$

resulting in a bottom intensified profile with vertical e-folding scale $\zeta$ set to $500 \mathrm{~m} . \mathrm{H}$ is the total depth.

Equation (2) was incorporated into the simulation so that $k_{v}$ (through its connection to the density field) is part of the model solution. Unlike Simmons et al. (2004), model $N_{b}$ was not used to update $E$ as the simulation progressed. We decided to avoid the required extrapolation of the near bottom buoyancy frequency due to the large uncertainty of the LGM deep ocean density field. The lack of $N_{b}$ also meant that $k_{v}$ could not be calculated for the bins touching the bottom. In these bottom bins diffusivity was set to be the same as in the bins above them.

The original background diffusivity $\left(k_{o}\right)$ of our initial experiments was, like in Simmons et al. (2004), set to $0.1 \mathrm{~cm}^{2} \mathrm{~s}^{-1}$. We were unable to obtain stable overturning in the LGM tidal mixing simulations with this lower value and this is why this parameter was changed to $0.2 \mathrm{~cm}^{2} \mathrm{~s}^{-1}$.

In (2), weak stratification ( small $\left.N^{2}\right)$ can lead to very large $\left(>>100 \mathrm{~cm}^{2} \mathrm{~s}^{-1}\right) k_{v}$ values. Based on a series of preliminary experiments, we opted to truncate $k_{v}$ at $5 \mathrm{~cm}^{2} \mathrm{~s}^{-1}$. This choice of maximum $k_{v}$ was a compromise between being able to represent areas with much larger than average mixing while still keeping the global diffusivity averages close to the canonical estimate of $1 \mathrm{~cm}^{2} \mathrm{~s}^{-1}$ (Munk 
(1966); Wunsch and Ferrari (2004)). It will be argued below that, while the truncation altered the global $k_{v}$ profiles (especially in the deeper layers), it did not cause other significant large scale changes.

A set of experiments was conducted using three depth dependent but horizontallyinvariant vertical diffusivity profiles based on Bryan and Lewis (1979) (BL). In what we define as the standard profile, $k_{v}$ varies from $0.6 \mathrm{~cm}^{2} \mathrm{~s}^{-1}$ in the first bin to near bottom values of about $1.3 \mathrm{~cm}^{2} \mathrm{~s}^{-1}$. This higher first bin diffusivity value results in a deeper, more realistic mixed layer in present day simulations with the model. There is no near surface maximum in what we define as the reduced surface mixing profile, which has a first bin value of $0.3 \mathrm{~cm}^{2} \mathrm{~s}^{-1}$. The reduced mid-depth profiles are standard profiles altered such that, at any depth, their values are smaller than the globally averaged tidal $k_{v}$ profile for the same sea level (Table 1, Fig.3, our definition of standard BL profile is based on the standard UVic ESCM configuration and some would categorize it as a BL profile with enhanced surface mixing.)

\subsection{Experiments}

Experiments were conducted under different climate states, sea level and mixing parameterizations (Table 1). The initial climate state was determined by prescribed radiative forcing and continental ice sheet distribution, with LGM referring to conditions at $\sim 21$ thousand years before present and PD to preindustrial conditions ( 1850). For the LGM simulations, wind anomalies based on surface temperature derived pressure fields were added to the reference winds (see Weaver et al. (2001)). 

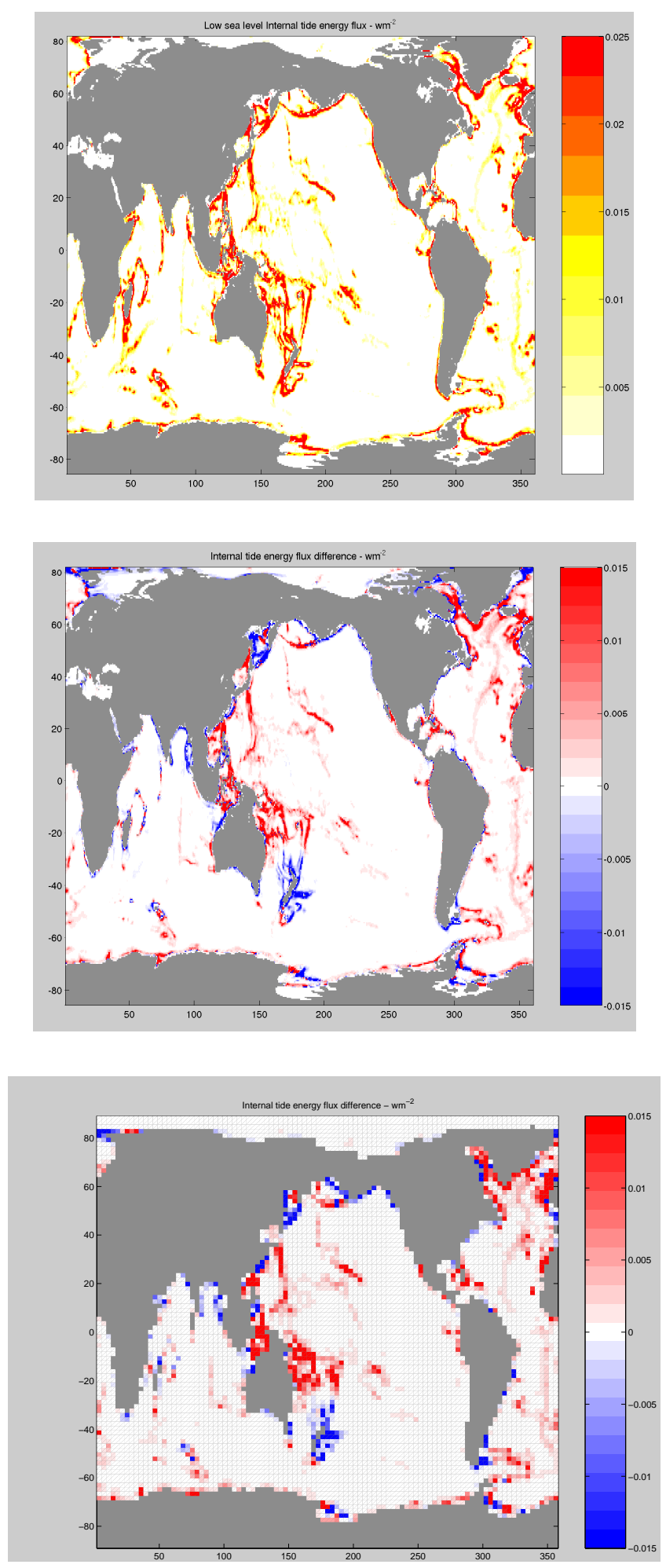

Fig. 1. Top, high resolution $\left(0.5^{\circ}\right)$ energy flux $(E)$ field used in the low sea level experiments; center, high resolution difference between low sea level and high sea level internal tide energy flux; bottom, same as center, interpolated to the UVic ESCM resolution. Values are in $\mathrm{Wm}^{2}$. 
The change in sea level in the UVic ESCM was performed by reducing the model's discretized bottom depths by $126 \mathrm{~m}$ and then re-binning the new depths into the same depth intervals adopted in the present day simulations. This assured that both low and high sea level experiments had the same depth discretization and facilitates comparisons between PD and LGM simulations. As the vertical extent of the bins increase toward the deeper layers, bottom bins that were originally at $1386 \mathrm{~m}$ (bottom of $9^{\text {th }}$ bin) were still discretized to $1386 \mathrm{~m}$ after having their value reduced to $1260 \mathrm{~m}$ (1386 m-126 m), because 1260 is closer to the original depth of $1360 \mathrm{~m}$ than to $1128 \mathrm{~m}$, the depth of the bottom of the $8^{\text {th }}$ bin. As a result, there was no change in the depth (and total number of vertical bins) in areas with bottom depth of $1386 \mathrm{~m}$ or deeper (Fig. 2) .

There were some significant differences, specially under LGM conditions, between experiments with the same BL type mixing but distinct sea levels (Table 2). This was taken as an indication that part of the model response to the change in sea level is not related to changes in the mixing. In order to isolate the impact of alterations to the deep ocean mixing from other consequences of sea level change, we conducted two tidal LGM experiments in which the ESCM sea level did not match the $E$ field (1). That is, a low/high sea level UVic ESCM simulation was performed using the $E$ field (1) obtained from the high/low sea level hydrodynamic tide model run.

Simulations were conducted until the model equilibrated, with equilibrium defined as trends in global ocean potential temperature and salinity smaller than $0.025^{\circ} \mathrm{K}$ and 0.0005 psu per thousand years. This usually required 4000 to 5000 years of model integration. 
Table 1

List of experiments. LGM stands for Last Glacial Maximum. PD (Present Day) refers to pre-industrial $(\sim 1850)$ climate state. "High" refers to present day sea level and "Low" to present day values minus $126 \mathrm{~m}$.

\section{Experiments}

\begin{tabular}{|c|c|c|c|c|}
\hline Name & Climate State & Sea Level & Mixing & other mixing changes \\
\hline Pd_hBL & $\mathrm{PD}$ & High & Bryan-Lewis & \\
\hline Pd_hBL_Rs & $\mathrm{PD}$ & High & Bryan-Lewis & reduced surface mixing \\
\hline Pd_hBL_Rm & $\mathrm{PD}$ & High & Bryan-Lewis & reduced mid-depth mixing \\
\hline Pd_lBL & $\mathrm{PD}$ & Low & Bryan-Lewis & \\
\hline Pd_hTd & $\mathrm{PD}$ & High & Tidal & \\
\hline Pd_lTd & $\mathrm{PD}$ & Low & Tidal & \\
\hline Lg_hBL & LGM & High & Bryan-Lewis & \\
\hline Lg_lBL & LGM & Low & Bryan-Lewis & \\
\hline Lg_hBL_Rs & LGM & High & Bryan-Lewis & reduced surface mixing \\
\hline Lg_hBL_Rm & LGM & High & Bryan-Lewis & reduced mid-depth mixing \\
\hline Lg_hTd & LGM & High & Tidal & \\
\hline Lg_lTd & LGM & Low & Tidal & \\
\hline Lg_hTd_lm & LGM & High & Tidal & low sea level tidal dissipation \\
\hline Lg_lTd_hm & LGM & Low & Tidal & high sea level tidal dissipation \\
\hline
\end{tabular}


High sea level

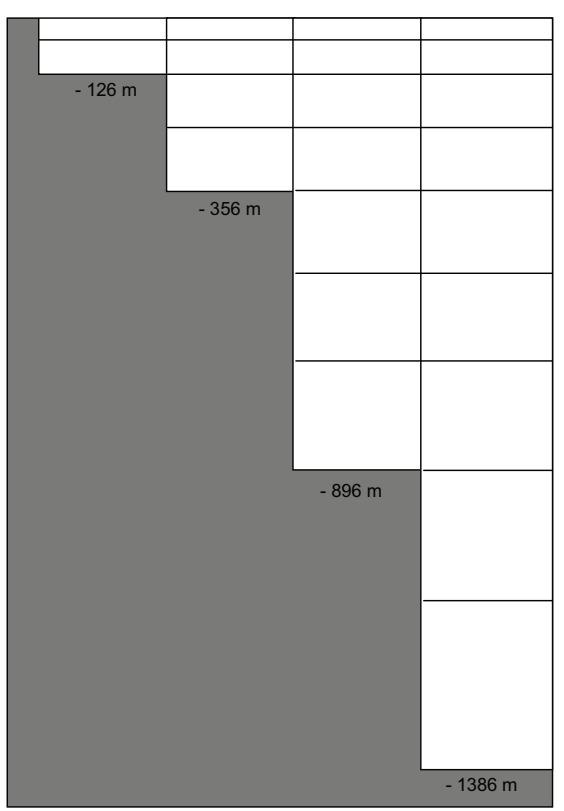

Low sea level

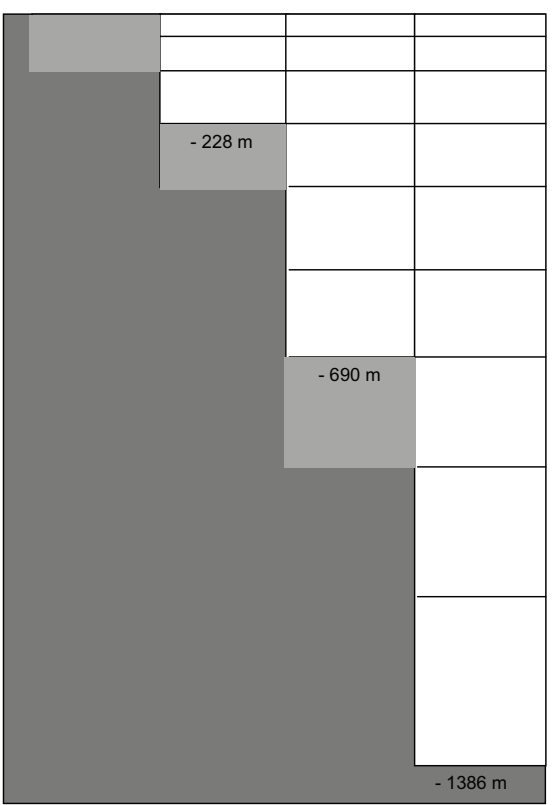

Fig. 2. Cartoon with example of how change in sea level was implemented. Values are depth in meters at the bottom bin. Relative size of bins is not to scale.Left, present day, high sea level configuration. Right, same set of bins for the LGM, low sea level configuration. The area with original depth of -126 m has been re-binned to land. The areas with original depths between $-1386 \mathrm{~m}$ and $-126 \mathrm{~m}$ have become shallower by one bin. The depth and number of bins in areas $-1386 \mathrm{~m}$ deep and depper remain the same.

\section{Results}

\subsection{Vertical diffusivity $\left(k_{v}\right)$}

The average diffusivities are larger in the tidal mixing simulations than in the BL experiments (Table 2). On average, tidal $k_{v}$ tends to be larger than BL 
$k_{v}$ throughout the water column, with the difference increasing in the deeper layers. The exception are the depths between $\sim 2500 \mathrm{~m}$ and $\sim 3000 \mathrm{~m}$, where the BL profiles can have slightly greater values. The BL reduce mid-depth profiles were constructed a posteriori and forced to have $k_{v}$ values smaller than the tidal mixing experiments at all depths (Fig.3). The increase of internal tide energy flux caused by lowering sea level results in a general increase of $k_{v}$ in the tidal low sea level experiments particularly below $\sim 2000 \mathrm{~m}$.

In the tidal mixing experiments, global mean diffusivity and its vertical profile are sensitive to the choice of maximum $k_{v}$ cutoff. In simulations where $k_{v}$ was truncated at $10 \mathrm{~cm}^{2} \mathrm{~s}^{-1}$ (not shown), global mean diffusivity values ranged from $\sim 2.8$ to $\sim 2.5 \mathrm{~cm}^{2} \mathrm{~s}^{-1}$, with maximum verticle profile average values between $\sim 7$ and $\sim 8 \mathrm{~cm}^{2} \mathrm{~s}^{-1}$. In the experiments discussed below, with $k_{v}$ truncated at $5 \mathrm{~cm}^{2} \mathrm{~s}^{-1}$, global means were between $\sim 1.6$ to $\sim 1.8 \mathrm{~cm}^{2} \mathrm{~s}^{-1}$ with maximum vertical average profile values around 4 to $4.5 \mathrm{~cm}^{2} \mathrm{~s}^{-1}$.

\subsection{Temperature and salinity}

Comparison of the global temperature and salinity profiles of the PD experiments to the mean World Ocean Database (WOA) profiles (Conkright et al. (2002)) do not show a marked difference between the BL and tidal mixing runs. This is in contrast to the Simmons et al. (2004) OGCM results, in which the tidal mixing experiment showed improved water mass distribution, but in agreement with other ESCM simulations (Schmittner et al. (2005)). While the use of tidal mixing resulted in smaller temperature bias above 1000 m (Fig. 4), the overall root mean square errors in both potential temperature and salinity of the tidal run are larger than in their BL counterparts (Table 2). As the 
Table 2

$k_{v}$, global, volume averaged vertical diffusivity; MOC, meridional overturning circulation averaged over the last 500 years of the simulations; P, power consumption due to diapycnal mixing, T-rms, root mean square difference between the simulated and the Levitus global temperature profile; S-rms, same as T-rms for salinity.

Average Parameters

\begin{tabular}{|c|c|c|c|c|c|}
\hline Experiment & $k_{v}\left(\mathrm{~cm}^{2}\right)$ & $\mathrm{MOC}(\mathrm{Sv})$ & $\mathrm{P}(\mathrm{TW})$ & T-rms $\left({ }^{o} \mathrm{C}\right)$ & S-rms (PSU) \\
\hline Pd_hBL & 0.9228 & 22.82 & 1.55 & 0.7061 & 0.0784 \\
\hline Pd_hBL_Rs & 0.9204 & 22.54 & 1.37 & 0.7811 & 0.0735 \\
\hline Pd_hBL_Rm & 0.8894 & 22.91 & 1.55 & 0.7224 & 0.0791 \\
\hline Pd_lBL & 0.9228 & 22.04 & 1.46 & & \\
\hline Pd_hTd & 1.5818 & 21.64 & 1.12 & 0.8912 & 0.0882 \\
\hline Pd_lTd & 1.7525 & 21.45 & 1.02 & & \\
\hline Lg_hBL & 0.9228 & 19.91 & 1.32 & & \\
\hline Lg_lBL & 0.9228 & 17.06 & 1.22 & & \\
\hline Lg_hBL_Rs & 0.9204 & 19.30 & 1.18 & & \\
\hline Lg_hBL_Rm & 0.8801 & 20.08 & 1.33 & & \\
\hline Lg_hTd & 1.5604 & 13.69 & 0.96 & & \\
\hline Lg_lTd & 1.7111 & 16.51 & 0.85 & & \\
\hline Lg_hTd_lm & 1.6814 & 13.79 & 1.00 & & \\
\hline Lg_lTd_hm & 1.5844 & 16,31 & 0.85 & & \\
\hline
\end{tabular}



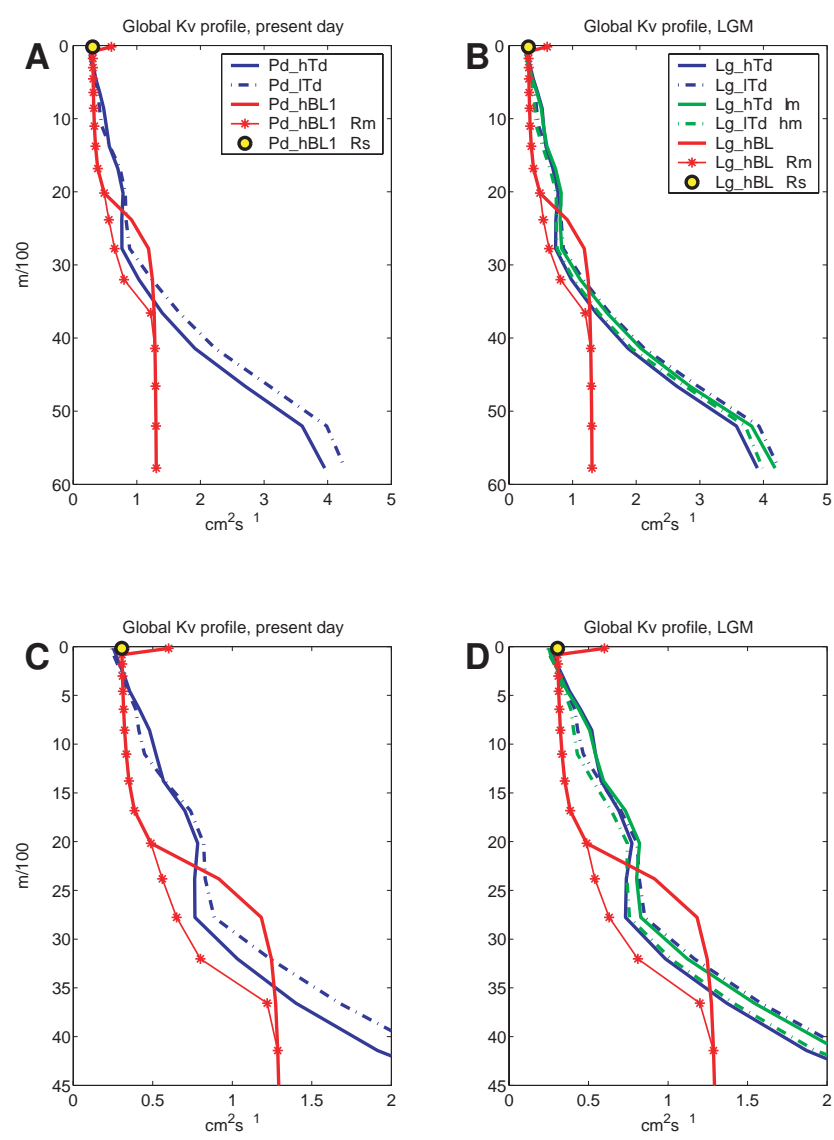

Fig. 3. PD (A) and LGM (B) vertical diffusivity $\left(k_{v}\right)$ profiles. Thick red, standard Bryan-Lewis (BL); thin red, mid-depth reduced BL; yellow dot represents the only change between the standard and surface reduced BL; solid blue, high seal level tidal mixing; dashed blue, low sea level tidal mixing; solid green, high seal level ESCM with low sea level $E$ tidal mixing; dashed green, low sea level ESCM with high sea level $E$ tidal mixing. C, same as A for the first $4500 \mathrm{~m} ; \mathbf{D}$, same B for the first $4500 \mathrm{~m}$

version of the UVic ESCM used in our experiments was optimized based on the adoption of BL mixing, the larger errors of the tidal simulations should be interpreted with caution and do not point to any intrinsic weakness of the tidal mixing scheme. 

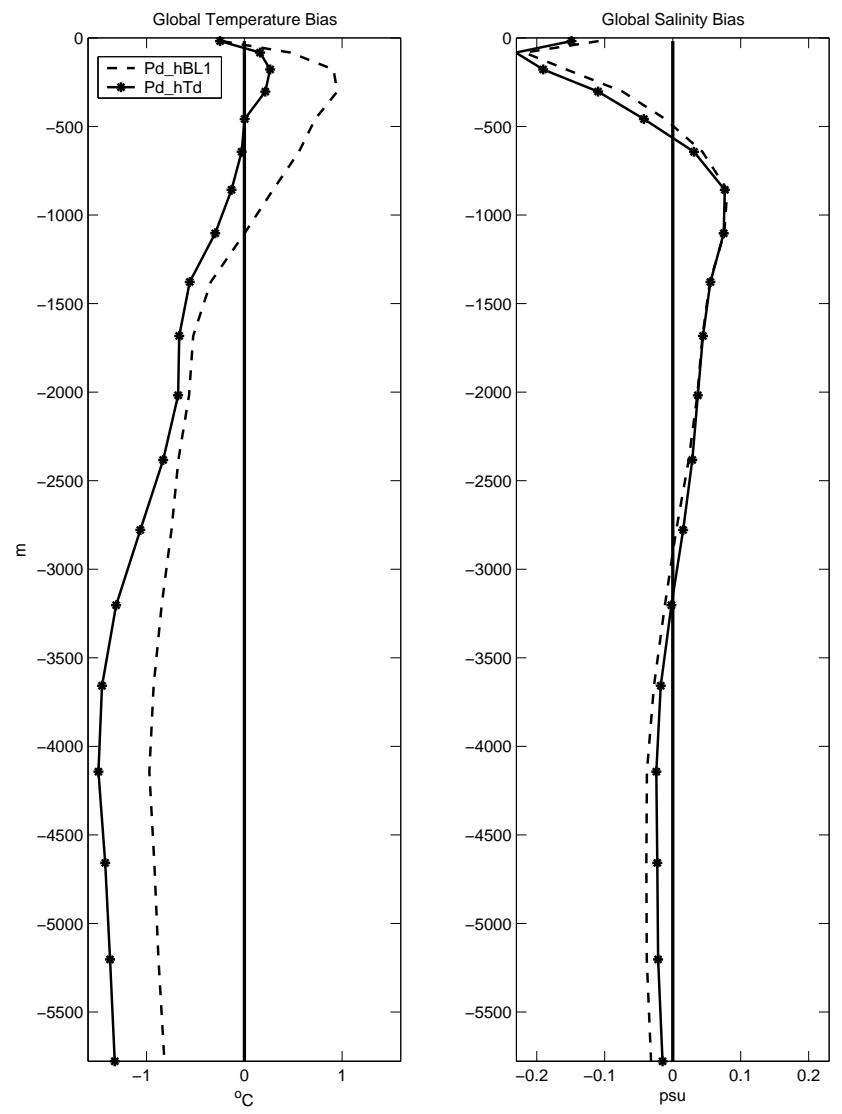

Fig. 4. Global, volume averaged potential temperature bias (left) and salinity bias (right) profiles (bias is defined as difference from the WOA climatology, Conkright et al. (2002)). See Table 1 for experiment description.

\subsection{Atlantic meridional overturning circulation (MOC)}

Under both PD and LGM conditions, tidal mixing experiments have lower MOC than the BL experiments, but the difference between the tidal experiments with higher MOC and the BL experiments with lower MOC is less than one Sv (Table 2). In all simulations, the spatial structure of the meridional overturning streamfunctions (not shown) remain essentially the same.

Unlike in simulations with constant mixing ((Bryan (1987); Manabe and Stouffer (1999); Schimittner and Weaver (2001)), a simple positive correlation be- 
tween average diffusivity and overturning transport is not observed in models with horizontally varying $k_{v}$ (Scott and Marotzke (2002); Saenko (2006); Simmons et al. (2004)). The same is the case in our results, where most tidal simulations have lower MOC but larger diffusivity than their BL equivalents.

No correlation exists either between $k_{v}$ and MOC in the tidal runs. This lack of correlation between MOC and global $k_{v}$ is also observed in comparisons between BL models with different mixing profiles. The change in global mean $k_{v}$ between the standard and mid-depth reduced BL experiments was larger than the mean $k_{v}$ change between the standard and the surface reduced BL simulations. In spite of its smaller effect on averaged mixing, the reduction in surface $k_{v}$ had a larger impact on the MOC than the reduction at mid-depth.

Further indication that global $k_{v}$ is not related in any simple way to MOC comes from tidal simulations with maximum vertical diffusivity truncated at $10 \mathrm{~cm}^{2} \mathrm{~s}^{-1}$. The mean $k_{v}$ of these experiments is on average $60 \%$ larger than the $k_{v}$ of simulations with diffusivity truncated at $5 \mathrm{~cm}^{2} \mathrm{~s}^{-1}$, while their MOC is about $1 \%$ lower than the ones with the smaller truncation value.

In OGCMs and UVic ESCM simulations with simpler configuration, meridional overturning was shown to be well correlated to an estimate of the power consumed by vertical mixing $(P)$, defined by the volume integral:

$$
P=\frac{1}{\Gamma} \int \rho k_{v} N^{2} d V
$$

Due to its dependency on stratification, most power is consumed in the first $1000 \mathrm{~m}$ of the water column. While our results show a general tendency for a positive correlation between MOC and global $P$ in the first $1000 \mathrm{~m}$, there is no clear relationship between the two parameters when all experiments are 
considered at once (Fig.5, top). There is more coherence in the relationship when particular climate states or sea levels are analyzed separately, especially for the BL experiments.

Numerical experiments under simplified conditions by Scott and Marotzke (2002) indicate that mixing at the boundaries is a more efficient driver of MOC strength than interior mixing. In an attempt to verify if the MOC could be better related to the power consumed by mixing in particular portions of the ocean, values of $P$ were estimated for the global ocean interior, the global ocean boundaries, the global ocean eastern boundaries and the global ocean western boundaries. In a further subdivision, $P$ was calculated for the upper $1000 \mathrm{~m}$ and the deep ocean for each of the classes above. In a final step, $P$ was estimated using all of the above categories for each separate ocean basin. None of these sub regions show an unequivocal relation between $P$ and MOC under all climate states and sea levels, including the western boundary, where results are very similar to the global comparison (Fig.5, bottom). The correlation between $\mathrm{MOC}$ and regional $k_{v}$ (separated in the same manner as $P)$ shows no relationship between the parameters.

One possible explanation for this lack of MOC response to $P$ is that the range of thermocline $k_{v}$, and consequently $P$, covered by the experiments is too small. In Simmons et al. (2004), for example, there is an near six fold increase in $P$ between their experiments with variable and constant mixing. In our experiments, the difference between the lowest and highest $P$ values for both tidal and BL simulations is about 30\%. There are indications that for the tidal mixing simulations, even if larger values of thermocline $k_{v}$ were obtained (by changes in $F$, for example), the impact on MOC would still be small. In experiments with spatially variable mixing by Saenko (2006), an order of 
magnitude increase in mean pycnocline $k_{v}$ from $\mathrm{O}\left(0.1 \mathrm{~cm}^{2} \mathrm{~s}^{-} 1\right)$ to $\mathrm{O}\left(1 \mathrm{~cm}^{2} \mathrm{~s}^{-} 1\right)$ resulted in about a $35 \%$ increase in $P$ and very little change to the MOC.

In simulations with variable mixing parameterizations (like Saenko (2006) and our tidal experiments) a large portion of the diffusivity is concentrated in very small areas of the ocean. Part of this lack of MOC response to increases in mean pycnocline diffusivity under these conditions can be explained by the fact that the efficiency with which these $k_{v}$ "hot spots" actually mix the water column is not proportional to the diffusivity. Indications of this can be seen by analyzing the changes in diffusivity and stratification between two PD high sea level model runs with very distinct truncations for maximum $k_{v}\left(10 \mathrm{~cm}^{2} \mathrm{~s}^{-1}\right.$ and $\left.100 \mathrm{~cm}^{2} \mathrm{~s}^{-1}\right)$. We compared areas that in both runs exhibited high mixing and that were shallow enough so that values of $k_{v}$ much higher than the background occurred in the pycnocline. Given the form of $F(z)$, this usually meant regions with depths of $500 \mathrm{~m}$ or less. In the mid- to low-latitude mixing "hot spots", the run with diffusivity truncated at $100 \mathrm{~cm}^{2} \mathrm{~s}^{-1}$ had $k_{v}$ values that were $30 \%$ to $50 \%$ higher than the experiment where maximum diffusivity was set to $10 \mathrm{~cm}^{2} \mathrm{~s}^{-1}$ while the equivalent change in buoyancy frequency $\left(N^{2}\right)$ was only in the order of $-0.5 \%$ to $-3 \%$. In the high latitudes (above $50^{\circ}$ ), this disparity is even greater, with 3 to 4 fold increases in $k_{v}$ generating alterations in average $N^{2}$ of less than $0.5 \%$.

Regarding the main question addressed by the simulations, while the extra internal tide energy flux under low sea level conditions did increase the overall vertical diffusivity, this increase did not cause a significant change in modelled $P$ and MOC values. This lack of response is seen in both PD and LGM experiments, which have very distinct rates of deepwater formation. It is clear that the MOC, particularly under the LGM climate state, is sensitive to sea 
level, but this connection is not primarily controlled by the amount of energy available for mixing. This is further illustrated by the LGM simulations with same sea level and distinct $E$ s, which allow the effects of mixing and sea level to be isolated. The reduction is sea level alone is associated with a $\sim 20 \%$ increase in MOC. The increase in tidal energy flux by itself results in a $\sim 1 \%$ increase in MOC.

Evidently, as with the maximum $k_{v}$ truncation value, other parameters from the tidal mixing scheme can influence the diffusivity and its potential impact on the MOC. For example, it has been shown that changes to the vertical structure of $k_{v}$, determined by the e-folding scale $\zeta$, will alter the amount of mixing in the thermocline and effect the MOC (Saenko (2006)). Also potentially important are $q$, the tidal dissipation efficiency, and $\Gamma$, the mixing efficiency. While we have kept these three parameters fixed during the simulations, we have explored the effects of a large range of $k_{v}$ truncation values which produced results that were similar to the ones that would be obtained by changes in the other parameters, particularly $\zeta$. This can be see in the changes in thermocline $k_{v}$ due to changes in truncation value described above.

The MOC for both BL and tidal mixing simulations was more sensitive to sea level changes under LGM than under PD climate state. Other than this, we find no overall coherent MOC response to changes in sea level. In the BL experiments, low sea level MOC is weaker than high sea level MOC. This is also the case for the PD tidal experiments but opposite to what is registered in the LGM tidal simulations. 

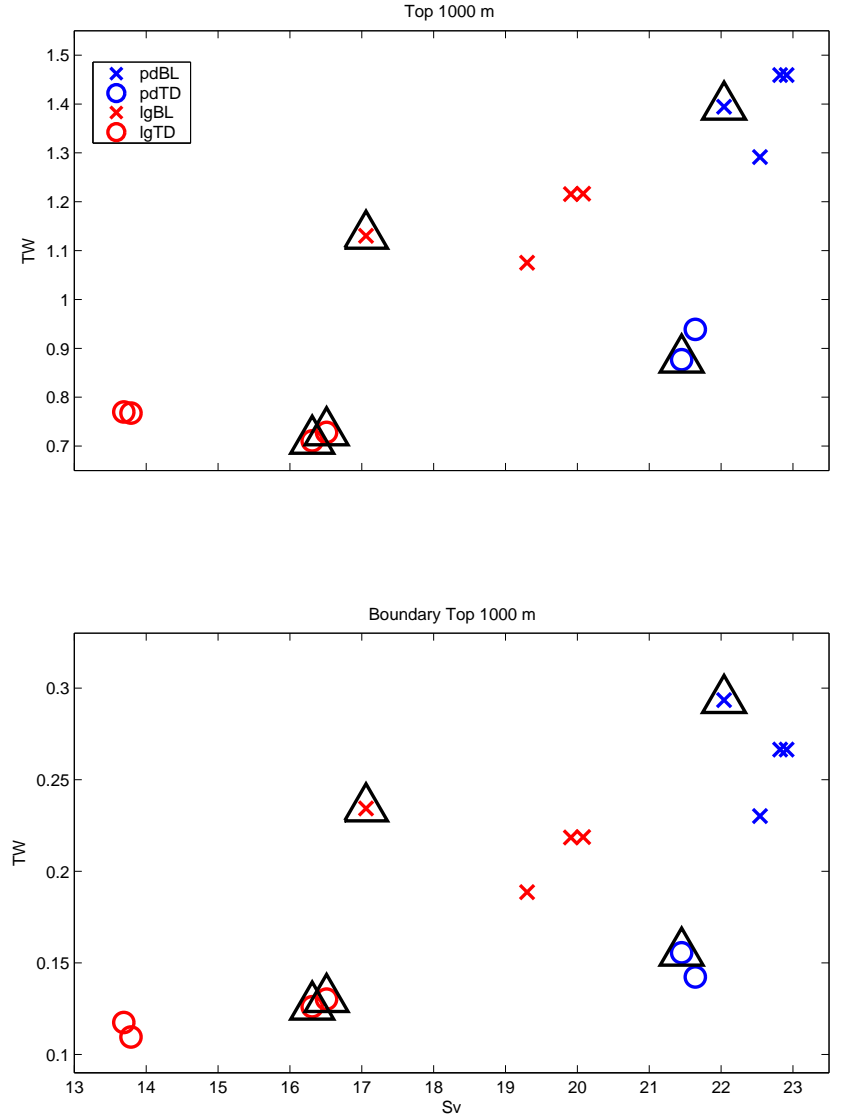

Fig. 5. Power consumption due to diapycnal mixing in the top $1000 \mathrm{~m}$ of the water column as a function of MOC. Top: whole world ocean; bottom: western boundaries. Triangles refer to low sea level simulations.

\subsection{Sensitivity to sea level change}

The disparity between BL and tidal response to sea level change make it impossible to determine a general relation between the isolated effects of sea level on the MOC. Still, the tidal LGM results show that the model's MOC response to sea level change alone is much larger than the response to alterations in tidal mixing under this climate state.

In the case of LGM ocean temperature and salinity, the model is clearly more sensitive to sea level variation than to changes in tidal mixing alone. The 
response of model surface air temperature to mixing is confined to the poles, but has similar magnitude to the changes associated exclusively with sea level (Fig.6, salinity not shown).

Interpretation of these results should take into account the fact that LGM $E$ was calculated using the present day bottom $N^{2}$ and that $E$ was not updated during the simulations. The option of not estimating $E$ during the experiments was motivated by the large uncertainty associated with the LGM deep density field.

The response of the model to sea level changes is complex and sensitive to climate state and choice of mixing parameterization. The goal of the simulations was to verify the effects of sea level on tidal mixing, particularly under a representative LGM climate state, and our experiments were not designed to analyze other potential impacts of sea level change. We opt to point out these interesting effects in order to compare them to isolated effects of tidal mixing but more a detailed discussion about them are beyond the scope of the project.

\section{Summary}

The increase in internal tide energy flux caused by a $\sim 120 \mathrm{~m}$ reduction in sea level had a clear effect on vertical diffusivity values, which were higher under low sea level conditions. The impacts of this change in diffusivity on the MOC are not straightforward and no clear relationship between diffusivity and overturning was found under PD or LGM climate sates. There is a weak positive correlation, for both tidal and BL mixing schemes, between MOC and 

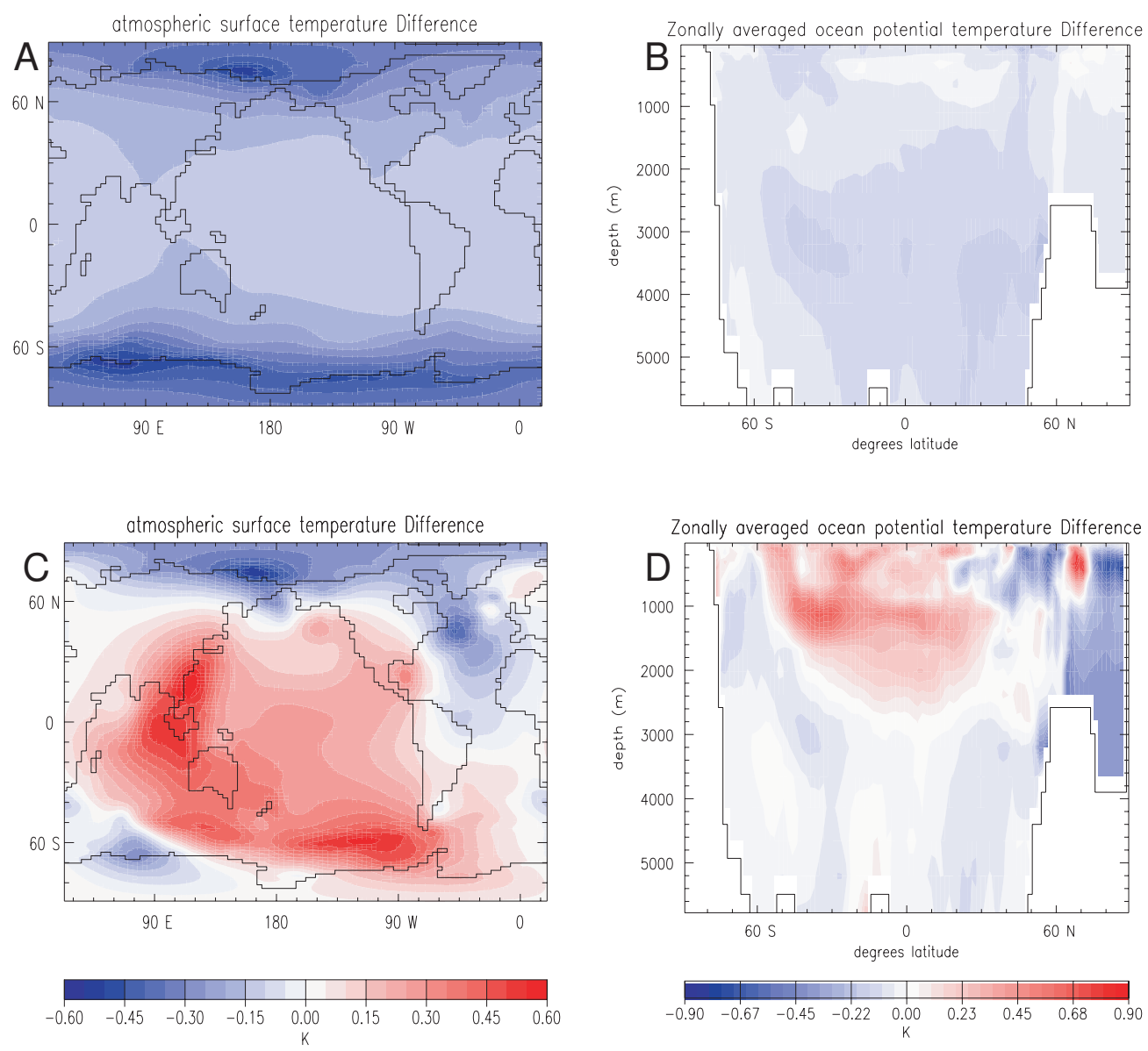

Fig. 6. Top row - differences between LGM simulations with same sea level but different tidal energy flux fields (Lg_lTd_hm - Lg_lTd). A, surface air temperature; B, zonally average ocean temperature. Bottom row - Same as top row but for simulations with same $E$ and different sea levels (Lg_hTd_lm - Lg_lTd). C, surface air temperature; D, zonally average ocean temperature.

the power consumed by vertical mixing.

While alteration in sea level caused significant changes to variables such as surface air temperature and ocean density, a large portion of this variation was not related to changes in tidal mixing. To capture the effects of sea level change in the LGM, simulations should account explicitly for the sea level reduction and not only take into consideration its impact on tide induced mixing. 


\section{Aknowledgements}

\section{References}

Bryan, F., 1987. Parameter sensitivity of primitive equation ocean generalcirculation models. J. Phys. Oceanography 17, 970-985.

Bryan, K., Lewis, L., 1979. A water mass model of the world ocean. Journal of Geophysical research 84, 2503-2517.

Conkright, M. E., Antonov, J. I., Baranova, O., Boyer, T. P., Garci, H. E., Gelfeld, R., Johnson, D., Locarnini, R. A., Murphy, P. P., O’Brien, T. D., Smolyar, I., Stephens, C., 2002. World ocean database 2001, volume 1: Introduction. ed: S. levitus, noaa atlas, nesdis 42. Tech. rep., U.S. Government Printing Office, Washington, D.C., 167 pp.

Egbert, G. D., Ray, R. D., 2000. Significant dissipation of tidal energy in the deep ocean inferred from satellite altimeter data. Nature 405, 775-778.

Egbert, G. D., Ray, R. D., Bills, B. G., 2004. Numerical modelling of the global semidiurnal tide in the present day and last glacial maximum. Journal of Geophysical Research 108, doi:10.1029/2003JC001973.

Gent, P., McWilliams, J., 1990. Isopycnal mixing in ocean general circulation models. Journal Physical Oceanography 20, 150-155.

Gent, P., Willebrand, J., McDougall, T. J., McWilliams, J. C., 1995. Parameterizing eddy-induced tracer transports in ocean circulation models. Journal Physical Oceanography 25, 463-474.

Holland, M. M., Bitz, C. M., Eby, M., Weaver, A. J., 2001. The role of iceocean interactions in the variability of the North Atlantic thermohaline circulation. J. Climate 14 (5), 656-675.

Holland, M. M., Brasket, A. J., Weaver, A. J., 2000. The impact of rising 
atmospheric CO2 on simulated sea ice induced thermohaline circulation variability. Geophysical Research Lett. 27 (10), 1519-1522.

Jayne, S. R., St. Laurent, L. C., 2001. Parameterizing tidal dissipation over rough topography. Geophysical Research Lett. 28, 811-814.

Ledwell, J., Montgomery, E., Polzin, K., Laurent, L., Schimitt, R., Toole, J., 2000. Mixing over rough topography in theBrazil Basin. Nature 403, 179182.

Manabe, S., Stouffer, R. J., 1999. Are two modes of thermohaline circulation stable? Tellus Series A-dynamic Meteorology Oceanography 51, 400-411.

Meissner, K. J., Weaver, A. J., Matthews, H. D., Cox, P. M., 2003. The role of land-surface dynamics in glacial inception: A study with the UVic Earth System Model. Climate Dynamics 21, 515-537.

Moum, J., Caldwell, D., Nash, J., Gunderson, G., 2002. Observations of boundary mixing over the continental slope. Journal of Physical Oceanography 32, $2113-2130$.

Munk, W., 1966. Abyssal recipes. Deep Sea Research 13, 707-730.

Nash, J. D., Kunze, E., Toole, J. M., Schmitt, R. W., 2004. Internal tide reflection and turbulent mixing on the continental slope. J. Phys. Oceanography $34,1117-1134$.

Pacanowski, R., 1996. Mom 2, version 2.0 Beta. Documentation, User's Guide and Reference Manual. Tech. rep., GFDL Ocean Technical report 3.2, Princeton, NJ.

Peltier, W. R., 2004. Global glacial isostasy and the surface of the ice-age earth: the ice-5g (VM2) model and GRACE. Annual Review of Earth and Planetary Sciences 32, 111-149.

Saenko, O. A., 2006. The effect of localized mixing on the ocean circulation and time-dependent climate change. J. Phys. Oceanography 36, 140-160. 
Schimittner, A., Weaver, A., 2001. Dependence of multiple climate states on ocean mixing parameters. Geophysical Research Letters 28, 1027-1030.

Schmittner, A., Meissner, K. J., Eby, M., Weaver, A. J., 2002. Forcing of the deep ocean circulation in simulations of the Last Glacial maximum. Paleoceanography 17.

Schmittner, A., Oschlies, A., Giraud, X., Eby, M., Simmons, H. L., 2005. A global model of the marine ecosystem for long-term simulations: Sensitivity to ocean mixing, buoyancy forcing, particle sinking, and dissolved organic matter cycling. Global Biogeochemical Cycles 19.

Scott, J. R., Marotzke, J., 2002. The location of diapycnal mixing and the meridional overturning circulation. J. Phys. Oceanography 32, 3578-3595.

Simmons, H. L., Jayne, S. R., St. Laurent, L. C., Weaver, A. J., 2004. Tidally driven mixing in a numerical model of the ocean general circulation. Ocean Modelling 6, 245-263.

Sloyan, B. M., 2005. Spatial variability of mixing in the Southern ocean. Geophysical Research Lett. 32.

St. Laurent, L., Simmons, H. L., Jayne, S. R., 2002. Estimating tidally driven mixing in the deep ocean. Geophysical Research Lett. 29.

Weaver, A. J., Eby, M., Wiebe, E., Bitz, C. M., Duffy, P. B., Ewen, T. L., Fanning, A. F., Holland, M. M., MacFayden, A., Matthews, H. D., Meissner, K. J., Saenko, O. A., Schmittner, A., Wang, Z., Yoshimori, M., 2001. The UVic Earth System Climate Model: Model description, climatology and application to past, present and future climates. Atmosphere-Ocean 39 (4), $361-428$.

Wunsch, C., Ferrari, R., 2004. Vertical mixing, energy and the general circulation of the ocenas. Annual Review of Fluid Mechanics 36, 281-314. 